CARDIOVASCULAR MEDICINE

\title{
Evaluation of a hand carried cardiac ultrasound device in an outpatient cardiology clinic
}

\author{
E C Vourvouri, D Poldermans, J W Deckers, G E Parharidis, J R T C Roelandt
}

Heart 2005;91:171-176. doi: 10.1136/hrt.2003.028225

\author{
See end of article for \\ authors' affiliations

...................... \\ Correspondence to: \\ Dr J R T C Roelandt, \\ Department of Cardiology, \\ Thoraxcentre-H 538, \\ Erasmus MC, $\mathrm{Dr}$ \\ Molewaterplein 40, \\ 3015 GD Rotterdam, \\ Netherlands: \\ j.r.t.c.roelandt@ \\ erasmusmc.nl
}

Accepted 5 March 2004

\begin{abstract}
Objective: To determine the diagnostic potential of a hand carried cardiac ultrasound (HCU) device (OptiGo, Philips Medical Systems) in a cardiology outpatient clinic and to compare the HCU diagnosis with the clinical diagnosis and diagnosis with a full featured standard echocardiography (SE) system. Methods: 300 consecutive patients took part in the study. The HCU examination was performed by an experienced echocardiographer before patients visited the cardiologist. The echocardiographer noted whether the HCU device was able to confirm or reject the referral diagnosis, which abnormality was detected, and whether SE investigation was necessary. Physical examination by a cardiologist followed and thereafter, whenever required, a complete study with an SE was carried out. The HCU data were compared with the clinical diagnosis of the cardiologist and the SE diagnosis in a blinded manner.

Results: The cardiologist referred 203 of 300 patients for an SE study and 13 patients for transoesophageal echocardiography. In 84 patients no further examination was considered necessary. $\mathrm{HCU}$ echocardiography was able to confirm or reject the suspected clinical diagnosis in 159 of 203 (78\%) patients. In 44 of 203 (22\%) patients SE Doppler was needed. Agreement between the HCU device and the SE system for the detection of major abnormalities was excellent (98\%). The HCU device missed $4 \%$ of the major findings. Among the 84 patients not referred for an SE, the HCU device detected unsuspected major abnormalities missed with the physical examination in 14 (17\%).

Conclusion: Integration of an HCU device with the physical examination augments the yield of information.
\end{abstract}

$\mathrm{T}$ he physical examination is the cornerstone of the evaluation of patients referred to the outpatient cardiology clinic, but often fails to provide a conclusive diagnosis. Since the introduction of echocardiography and Doppler, the limitations of the physical examination of specific cardiac abnormalities especially in their early stage have been shown. ${ }^{1}$ In addition, the auscultation skills and experience of cardiologists have declined due to less training in the curriculum as a result of the existing time pressure and the increasing availability and reliance on more sophisticated imaging methods. ${ }^{2-5}$ Consequently, echocardiography and Doppler are the initial diagnostic tests ordered for most of the patients. In practice, most of these tests are performed several days after the first patient-physician encounter, leading to a delay in the final diagnosis and sometimes treatment.

Recently, small hand carried cardiac ultrasound (HCU) devices have become available and initial studies have shown their validity in the immediate diagnosis of cardiac abnormalities at the point of care. ${ }^{6-10}$ HCU examination may differentiate normal from abnormal conditions and recognise patients with significant cardiac abnormalities that can be missed by physical examination alone.

The aim of the present study was to determine the diagnostic potential of an HCU device in patients referred for an initial visit to the outpatient cardiology clinic (new patients). The HCU diagnosis was compared with the diagnoses of the cardiologist's physical examination and of a full featured standard echocardiography (SE) examination.

\section{MATERIALS AND METHODS}

\section{Study population and design}

During a five month period, 300 new patients referred to the outpatient clinic of the Thoraxcentre, Rotterdam, were examined with an HCU device by an experienced echocardiographer (cardiologist) before the patient's initial visit to the cardiologist. Table 1 lists the patients' characteristics. The echocardiographer had the referral diagnosis for which the patient was sent to the cardiologist. She took a brief history, performed the HCU examination (maximum duration 10 minutes), and noted her findings together with a qualitative assessment and quantitative results. She then noted whether further echocardiography and Doppler examination with SE was needed. Subsequently, the patient was examined by the consulting cardiologist, who was unaware of the HCU evaluation and who decided whether an echocardiographic examination with an SE system (Sonos 5500, Philips Medical Systems, Eindhoven, the Netherlands; or System Five, Vingmed, Horten, Norway) was indicated. This examination was then performed by an independent echocardiographer (sonographer) who was blinded to the HCU results. A cardiologist not involved in the study interpreted the SE examination results. All patients had an ECG recorded as a routine clinical practice. The clinical diagnosis made by the physician was based on the combination of clinical history, physical examination, and ECG findings. The institutional medical ethics committee approved the study and informed consent was obtained from all patients.

\section{The HCU device}

The OptiGo (Philips Medical Systems) HCU device was used (fig 1). It is equipped with a $2.5 \mathrm{MHz}$ phased array broadband transducer and operates on a rechargeable lithium ion battery or alternating current. Two dimensional imaging, colour flow Doppler imaging, and two callipers for linear measurements are also integrated with the system. Images are documented on a CompactFlash card.

Abbreviations: $\mathrm{HCU}$, hand carried cardiac ultrasound; LV, left ventricular; SE, standard echocardiography 
Table 1 Baseline characteristics of 300 new patients referred to the outpatient cardiology clinic

\begin{tabular}{ll}
\hline Characteristic & $\mathbf{n}(\%)$ or mean (SD) \\
\hline Age (years) & $53(16)$ \\
Men & $57(19 \%)$ \\
Documented CAD & $61(20 \%)$ \\
Known hypertension & $86(29 \%)$ \\
Treated DM & $11(4 \%)$ \\
Previous valve repair & $4(1 \%)$ \\
Previous prosthetic valve & $3(1 \%)$ \\
\hline CAD, coronary artery disease; DM, diabetes mellitus.
\end{tabular}

\section{Echocardiographic diagnosis with the HCU device} Cardiovascular abnormalities were classified as major or minor. Major abnormalities were considered to be the clinically significant abnormalities that would trigger further diagnostic evaluation, to change or initiate treatment management, or to have prognostic significance (table 2). Minor abnormalities were considered to be abnormalities that were of no clinical significance and would therefore not affect decision making for patient management.

Valve or flow abnormalities detected with the HCU device were evaluated with the pulsed and continuous wave Doppler modalities of the SE. The severity of these abnormalities was graded according to standard methods. ${ }^{11}$

Unsuspected major abnormalities (see table 2 for definition of "major") were considered to be the echocardiographically detected major abnormalities that were not reported in the history or were not suspected or previously described from the symptoms of the patient. Furthermore, the cardiologist's physical examination missed these abnormalities.

\section{Statistical analysis}

Descriptive statistics are reported as mean (SD) for continuous variables and as percentages for categorical variables. The agreement between the two examination techniques for the detection of major abnormalities was assessed from $2 \times 2$ tables with weighted $\kappa$ statistics. On the basis of Fleiss's classification, $\kappa$ values $<0.4$, between 0.4 and 0.75 , and $>0.75$ were considered to indicate poor, fair to good, and excellent agreement, respectively. ${ }^{12}$

\section{RESULTS}

\section{General results}

Table 3 presents the most common referral question or suspected diagnosis for the 300 patients sent by the primary care physician to the cardiology outpatient clinic. The cardiologist requested after his physical examination an echocardiographic examination for 203 of the 300 (68\%) patients. Table 3 also lists the most common referral question or suspected clinical diagnosis for which the SE was requested in these 203 patients. Thirteen of 300 (4\%) patients were sent for a transoesophageal study for the evaluation of a cardiac source of embolism. No further examination was considered necessary for 84 of the 300 (28\%) patients.

\section{Patients sent for an SE by the cardiologist}

The HCU examination was able to confirm or reject the clinical diagnosis of the cardiologist in 159 of the 203 (78\%) patients. For example, a suspected clinical diagnosis of left ventricular (LV) hypertrophy can be easily and reliably confirmed or rejected with an HCU examination without the need for SE. ${ }^{8}$

In 44 of $203(22 \%)$ patients the HCU device was able to confirm or reject the clinical diagnosis, but haemodynamic

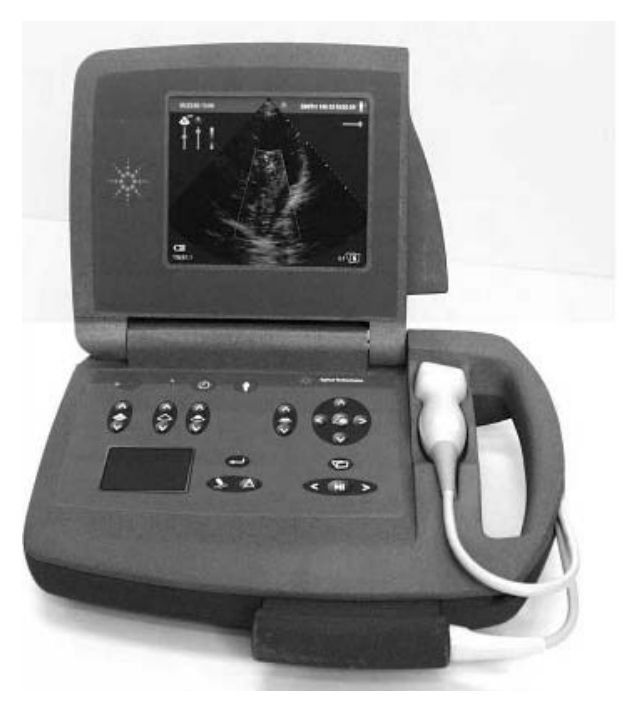

Figure 1 The OptiGo device, the hand carried cardiac ultrasound (HCU) device used in the study.

Table 2 Major cardiovascular findings

\begin{tabular}{ll}
\hline Finding & $\begin{array}{l}\text { Grade constituting major } \\
\text { abnormality }\end{array}$ \\
\hline Valve regurgitation & $>$ Mild \\
Valve stenosis & Any \\
LV dysfunction & LVEF $<45 \%$ \\
RV dysfunction* & Any \\
LV hypertrophy† & $>14 \mathrm{~mm}$ \\
Mitral valve prolapse & Any \\
Congenital abnormality & Any \\
Pericardial effusion & Any \\
Vegetation & Any \\
Mass lesion & Any \\
\hline
\end{tabular}

*Normal right ventricular (RV) systolic function was defined by normal RV dimensions (end diastolic transverse diameter $\leqslant 45 \mathrm{~mm}$ ) in the four chamber view ${ }^{11}$; tleft ventricular (LV) hypertrophy was defined as a major abnormality when the septal wall thickness was $>14 \mathrm{~mm}$.

LVEF, left ventricular ejection fraction.

Doppler assessment was regarded as necessary. Since this feature was not available on the HCU an SE examination followed for the evaluation of the following findings: severity of valve stenosis (14 patients); valvar regurgitant lesion (10 patients); diastolic LV function (four patients); LV outflow tract obstruction (seven patients); congenital abnormality (six patients); and prosthetic valve evaluation (three patients). Of note, in all of these patients the valvar or congenital lesion was detected and a gross estimation of their severity was possible with the HCU device.

\section{Patients not sent for SE by the cardiologist}

The cardiologist did not refer 84 of $300(28 \%)$ patients for an SE study. In $17 \%$ (14 of 84 ) of these patients, a major unsuspected abnormality missed by the physical examination was detected with the HCU device. Furthermore, the HCU device detected no abnormalities in 17\% ( 14 of 84 ) and minor abnormalities in $66 \%$ (56 of 84 ).

The HCU echocardiographer noted that an SE examination would be necessary for $14 \%$ ( 12 of 84 ) of this group of patients because the Doppler modality was needed (three patients were from the group with unsuspected major abnormalities). 
Table 3 Reasons for referral by primary care physicians and cardiologists

\begin{tabular}{ll}
\hline Possible diagnosis/question & Proportion \\
\hline Referral to outpatient cardiology clinic by primary & \\
care physician (300 patients) & \\
LV dysfunction & $31 \%$ \\
Preoperative cardiac evaluation & $15 \%$ \\
Rhythm abnormalities & $14 \%$ \\
Cardiac source of embolism & $8 \%$ \\
Family history & $7 \%$ \\
LV hypertrophy & $5 \%$ \\
Murmur evaluation & $5 \%$ \\
Dyspnoea, fatigue, dizziness & $4 \%$ \\
Congenital abnormality & $4 \%$ \\
Miscellaneous & $6 \%$ \\
Referral to the echocardiographic laboratory by & \\
the cardiologist (203 patients) & \\
LV dysfunction & $59 \%$ \\
Valve evaluation & $27 \%$ \\
LV hypertrophy & $11 \%$ \\
Congenital abnormality & $7 \%$ \\
Mitral valve prolapse & $4 \%$ \\
Prosthetic valve evaluation & $2 \%$ \\
Endocarditis & $2 \%$ \\
RV dysfunction & $1.5 \%$ \\
Thrombus & $1 \%$ \\
\hline The preoperative patient group had either symptoms or a history of \\
cardiac disease.
\end{tabular}

\section{Major and minor abnormalities detected with the HCU device and the SE system}

In total 217 patients had an SE examination. The cardiologist referred 203 patients and the HCU examiner independently referred 14 patients.

The agreement between the two devices for the detection of major abnormalities was excellent $(98 \%, \kappa=0.95)$ (table 4). Table 5 lists the abnormalities detected by the SE system and missed by the HCU device and the physical examination. The HCU device missed six major abnormalities: a small ventricular septal defect that was located high, two aortic stenosis (one moderate with a peak gradient of $40 \mathrm{~mm} \mathrm{Hg}$ and one mild with a peak gradient of $25 \mathrm{~mm} \mathrm{Hg}$ ), a moderate mitral and aortic regurgitation, both in the same patient with a poor echo window, and a moderate LV dysfunction.

Figure 2 gives a simple overview of the correlation of major and minor findings between the HCU device and the SE system.

\section{Unsuspected major findings}

In total, 71 unsuspected major findings were detected echocardiographically in 61 of 300 patients $(20 \%)$. Importantly, of these 71 findings 15 were present in 14 of the $84(17 \%)$ patients not referred to SE by the cardiologist. In particular, these findings were LV hypertrophy (six patients), hypertrophic obstructive cardiomyopathy (one patient), LV dysfunction or wall motion abnormalities (three patients), valvar regurgitation (two patients), dilated ascending aorta (one patient), mitral valve prolapse (one patients), and pericardial effusion (one patient). Two of these findings were detected in the same patient. Three patients needed SE Doppler examination for gradient evaluation after the HCU examination. Physical examination misdiagnosed hypertrophic obstructive cardiomyopathy as aortic stenosis in one patient.

The 71 unsuspected major findings were the following: LV hypertrophy (28 patients), valvar regurgitation (20 patients), valvar stenosis (six patients), LV dysfunction (five patients), hypertrophic cardiomyopathy (four patients), and miscellaneous (eight patients).
Table 4 Agreement of detection of major abnormalities in 217 patients between the OptiGo and a standard echocardiography system

\begin{tabular}{lllc}
\hline & \multicolumn{2}{c}{ SE system } & \\
\cline { 3 - 4 } & & Abnormal & Normal \\
\hline \multirow{2}{*}{ HCU device } & Abnormal & 92 & 0 \\
& Normal & 5 & 120 \\
\hline
\end{tabular}

Data are the absolute number of patients.

Agreement $98 \%, \kappa=0.95$, sensitivity $95 \%$ (95\% confidence interval (CI) 0.91 to 0.91$))$, specificity $100 \%(95 \% \mathrm{Cl} 0.97$ to 0.97$)$, positive predictive value $100 \%$ ( $95 \% \mathrm{Cl} 0.96$ to 0.96 ), negative predictive value $96 \%$ (95\% Cl 0.93 to 0.93 ).

$\mathrm{HCU}$, hand carried cardiac ultrasound; normal, no abnormalities or minor abnormalities; SE, standard echocardiography.

Table 5 Comparison between the SE system and the $\mathrm{HCU}$ device in the detection of minor and major cardiovascular findings in 203 patients sent for an SE examination

\begin{tabular}{lccc}
\hline & SE: finding & $\begin{array}{l}\text { HCU } \\
\text { device: } \\
\text { finding } \\
\text { missed }\end{array}$ & $\begin{array}{l}\text { Physical } \\
\text { exam: } \\
\text { finding } \\
\text { missed }\end{array}$ \\
\hline Finding & 31 & 7 & NA \\
\hline Minor findings & 70 & 13 & NA \\
Aortic regurgitation & 49 & 18 & NA \\
Mitral regurgitation & $150^{*}$ & 38 & NA \\
Tricuspid regurgitation & 14 & 2 & 4 \\
Total & 10 & 1 & 5 \\
Major finding & 3 & 0 & 2 \\
Aortic stenosis & 18 & 1 & 7 \\
Aortic regurgitation & 6 & 0 & 5 \\
Mitral stenosis & 1 & 0 & 0 \\
Mitral regurgitation & 34 & 1 & 2 \\
Tricuspid regurgitation & 1 & 0 & 0 \\
Pulmonary stenosis & 29 & 0 & 23 \\
LV systolic dysfunction & 5 & 0 & 3 \\
RV systolic dysfunction & 4 & 1 & 1 \\
LV hypertrophy & 3 & 0 & 0 \\
Mitral prolapse & 3 & 0 & 1 \\
Ventricular septal defect & 7 & 0 & 3 \\
Atrial septal defect & 138 & 6 & 56 \\
Pericardial effusion & & & \\
HOCM & & & \\
Total & & & \\
\hline
\end{tabular}

Some patients had more than one cardiovascular abnormality. *The 150 minor findings were present in 107 patients; the 138 major findings were present in 83 patients. The physical examination missed 56 major findings (in 47 patients). Physical examination misdiagnosed moderate mitral regurgitation as aortic stenosis (false positive) in two patients and aortic stenosis as mitral regurgitation in one patient. HOCM, hypertrophic obstructive cardiomyopathy; NA, not applicable.

Figures 3, 4, and 5 present examples of major unsuspected findings detected with the HCU device and verified with the SE system.

\section{DISCUSSION}

The first HCU device to augment the physical examination was introduced by Roelandt and colleagues in Rotterdam in 1978. ${ }^{13-15}$ However, the combination of the poor image quality, technical limitations, and reimbursement issues discontinued its development. Today, advances in microprocessor technology have led to a new generation of portable echocardiographic systems with excellent image quality and significantly lower cost than SE machines. Several studies have shown their efficacy and accuracy for the diagnosis of major abnormalities at the point of care. ${ }^{6} 10$ 


\begin{tabular}{lll|}
\hline & \\
$n=203$ & Referred for TOE & No additional echocardiogram requested \\
$83 / 203(41 \%)$ & $6 / 13(46 \%)^{\dagger}$ & $n=84$ \\
$78 / 203(38 \%)$ & $5 / 13(38 \%)$ & $14 / 14^{\ddagger}$ \\
$107 / 203(53 \%)$ & $3 / 13(23 \%)^{\dagger}$ & $14 / 84(17 \%)$ \\
$91 / 203(45 \%)$ & $5 / 13(38 \%)$ & $10 / 14^{\ddagger}$
\end{tabular}

Agreement $\mathrm{HCU} / \mathrm{SE}=\mathbf{9 8} \%(\kappa=\mathbf{0 . 9 5})$

Patients with unexpected major findings with $\mathrm{HCU}=14 / 84$ (17\%)

Figure 2 Agreement between the hand carried cardiac ultrasound (HCU) device and standard echocardiography (SE) system was $98 \%(\kappa=0.95)$. HCU found unexpected major abnormalities in 14 of $84(17 \%)$ patients. *Absolute number of patients with abnormalities; tfindings from the transoesophageal echocardiography (TOE) examination; łof the 84 patients not referred for echocardiography by the cardiologist, 14 with major abnormalities on the HCU underwent SE examination after it was requested by the HCU echocardiographer.

In the current study in $78 \%$ of patients sent for a full featured SE examination by the cardiologist, the HCU device provided instant information potentially sufficient to avoid an SE examination. Compared with the SE system, the HCU device had a positive predictive value in diagnosing the major abnormalities of $100 \%$ and a negative predictive value of $96 \%$. Furthermore, an unsuspected major abnormality was found in $23 \%$ of these patients with both the HCU device and the SE system. These abnormalities were not expected based on the history and the patient's symptoms and were on top of the clinical referral diagnosis made by the cardiologist. The HCU device missed $4 \%$ of the major findings. However, it is obvious that knowing the results of the physical examination and the detailed clinical history beforehand would have led to a more focused echocardiographic examination for major

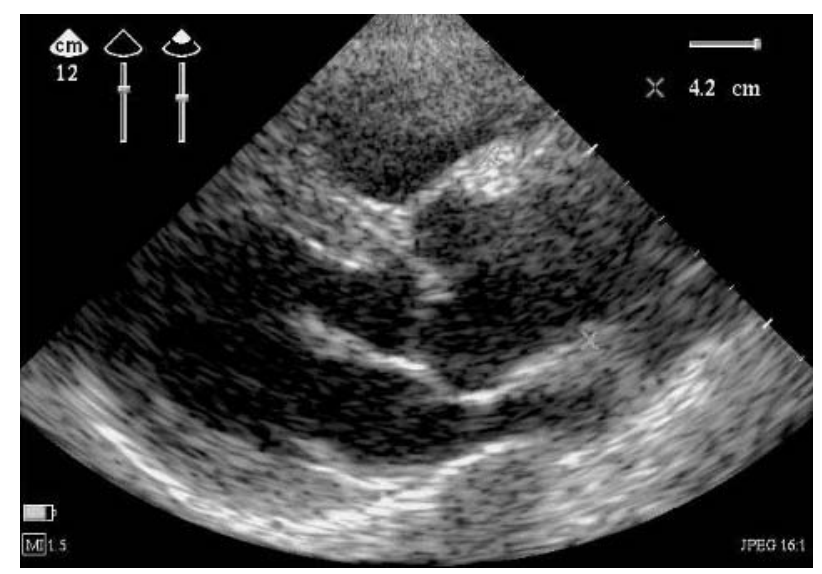

Figure 3 Parasternal long axis view of a 48 year old patient with a dilated ascending aorta $(42 \mathrm{~mm})$. The patient had a history of palpitations and was referred to the outpatient cardiology clinic for evaluation of atrioventricular nodal re-entry tachycardias. findings with high probability and would have reduced the number of missed findings by the HCU device.

Most important, in 84 of $300(28 \%)$ patients the cardiologist considered his physical examination sufficiently accurate to decide that echocardiographic assessment was unnecessary. However, in $17 \%$ of these patients unsuspected major abnormalities were found with the HCU device and missed by the physical examination. This is in concordance with the study of Fedson and colleagues. ${ }^{17}$ It is obvious that direct visualisation leads to a more accurate diagnosis than indirect observation by palpation and auscultation even by the best experts. "Seeing" enables the pre-symptomatic detection of abnormalities and of abnormalities that are beyond physical signs. It is generally recognised that physical examination has shortcomings and that the clinical diagnosis

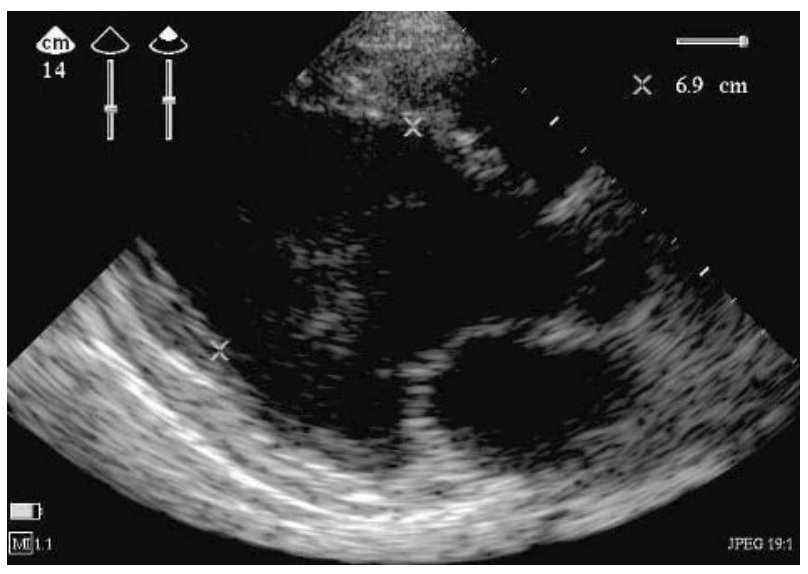

Figure 4 Parasternal long axis view of a 46 year old woman with a history of cancer. She was referred for preoperative evaluation. Left ventricular end diastolic dimension is $69 \mathrm{~mm}$. Ejection fraction was visually estimated to be $<35 \%$. The patient has dilated cardiomyopathy. 

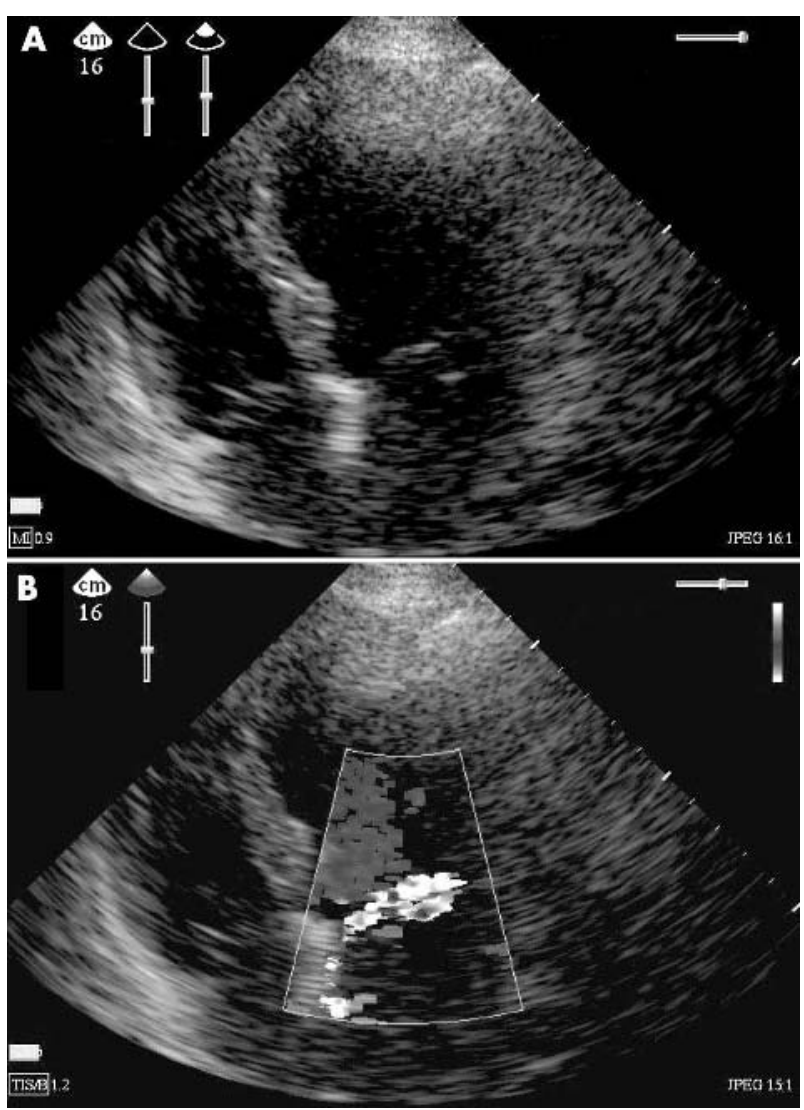

Figure 5 Apical four chamber view of a 45 year old patient with (A) prolapse of the posterior mitral leaflet and (B) an eccentric jet towards the interatrial septum. The patient was referred for evaluation of palpitations and was known to have a systolic murmur.

of many common cardiac conditions such as pericardial effusion, early LV dysfunction, hypertrophic cardiomyopathy, or silent valvar disease can challenge the most experienced clinicians, whereas they are readily diagnosed by echocardiography and Doppler examination. ${ }^{1}{ }^{18}$ The incorporation of HCU into the physical examination can undoubtedly provide physicians with instant valuable information assisting them in clinical diagnosis and patient management. In the current study the HCU device was tested in an outpatient cardiology clinic. The HCU device has been studied in various clinical settings. ${ }^{6}{ }^{10} 1920$

Patients referred to our outpatient clinic, which is a tertiary referral centre, have a high prevalence of cardiac disease, which explains the high number of requests for an echocardiographic examination. However, since outpatient echocardiographic examinations are often performed several days after the patient's first visit, the patient must return to the hospital for the examination. With the incorporation of HCU into the physical examination, this extra visit to the hospital could have been avoided by $78 \%$ of our study cohort for whom the cardiologist requested an SE examination. But even for the institutions where an SE examination is standard procedure after the physical examination, the integration of HCU with the physical examination can lead to significant cost and time savings, since the majority of referred patients have no abnormalities.

Our study is in concordance with the study of Spencer and colleagues, $^{21}$ who compared HCU echocardiography at the point of care with the physical examination in a smaller patient cohort (36 patients). They reported that the use of this device by cardiologists reduced the number of major cardiovascular findings missed by the clinical examination. Minor abnormalities were more easily missed. This is in agreement with our findings that the HCU device detected mild valvar regurgitations in only 112 of 150 patients. Furthermore, Galasko and colleagues ${ }^{19}$ studied 562 patients in a community setting with the same HCU device and correctly identified major abnormalities (LV dysfunction, LV hypertrophy, and left sided valvar regurgitation). They also concluded that minor disease is more likely to be missed.

Spectral flow Doppler is available on some of the HCU devices. Such devices have been shown to overcome limitations in evaluating haemodynamic variables ${ }^{22}$ and may limit referral for the full featured SE system even further. But still this needs to be evaluated in further studies.

\section{Training requirements}

There is no doubt that training is required to use an imaging device. The current study was performed by a cardiologist with expertise in both echocardiography and HCU devices (ECV). Training of clinicians and non-echocardiographers should focus on the criteria of identifying a normal heart and diagnosing major and acute cardiac disorders. The American Society of Cardiology ${ }^{23}$ has recently published guidelines regarding the use of such devices, recommending level I training as an absolute minimum..$^{24}$ However, recent studies have suggested that it is possible to train physicians and students to detect significant structural or flow abnormalities in a short period of time with high efficacy. ${ }^{25-29}$

Physical examination remains the cornerstone of cardiovascular assessment and its use should be encouraged. However, visualising the heart at the point of care helps us to differentiate normal from abnormal conditions and thus to reduce the number of normal referrals, leading to targeted referrals for further diagnostic assessment whenever regarded as necessary. The HCU device should be used as phonocardiography was used in the past: whenever documentation was needed after auscultation. Thus, in case of doubt or whenever haemodynamic and quantitative information is crucial for further management, an examination with a full featured SE system should follow the HCU examination. The appropriate training of clinicians to use such devices is the basic element needed for the future use of the HCU device in daily clinical practice and efforts should be made in this respect.

It should be remembered that the real value of any imaging technology is intimately dependent on our intellectual contribution and individual responsibility.

\section{Limitations}

The HCU device that was used for this study had no spectral Doppler modality to obtain haemodynamic data. The valvar abnormalities were evaluated qualitatively with the two dimensional and colour flow Doppler features. However, continuous wave and pulsed wave Doppler echocardiography is necessary for an accurate assessment of valvar regurgitation or stenosis. Furthermore, examination of transmitral flow gives information about the diastolic function and filling pressures. Since this was a blind study, the cardiologist was not aware of which patients had undergone an HCU examination. This was done to avoid any bias that would lead the cardiologist to change his potential request for an SE. Therefore, not all patients had an SE examination, but only when the cardiologist requested it. However, many studies have already proved the accuracy and reliability of the HCU device compared with the SE system in detecting cardiovascular abnormalities. Furthermore, the main interest is to compare the yield of the physical examination with versus without an HCU device. 


\section{Conclusion}

In patients who attend the outpatient cardiology clinic, integration of an HCU device with the physical examination allows the immediate detection of structural cardiac disease. Often a definitive diagnosis is made or the patient is referred for specific diagnostic tests. A major advantage is that patients with no cardiac abnormality can avoid further echocardiographic tests (negative predictive value is 96\%). It appears that the yield of the physical examination is augmented, which alone may miss significant abnormalities.

\section{Authors' affiliations}

E C Vourvouri, D Poldermans, J W Deckers, G E Parharidis,

J R T C Roelandt, Department of Cardiology, Thoraxcentre, Erasmus MC, Rotterdam, Netherlands

\section{REFERENCES}

1 Popp RL. The physical examination of the future: echocardiography as part of the assessment. ACC Curr Rev 1998;7:79-81.

2 Hu BS, Altiel F, Popp RL. Effectiveness of limited training in echocardiography for cardiovascular diagnosis. Circulation 1996;94(suppl):I253.

3 Mangione S, Nieman LZ. Cardiac auscultatory skills of internal medicine and family practice trainees: a comparison of diagnostic proficiency. JAMA 1997; 278:717-22.

4 Mangione $\mathbf{S}$. Cardiac auscultatory skills of physicians-in-training: a comparison of three English-speaking countries. Am J Med 2001;1 10:210-6. 5 Tavel ME. Cardiac auscultation: a glorious past-but does it have a future? Circulation 1996:93:1250-3.

6 Vourvouri EC, Poldermans D, de Sutter J, et al. Experience with an ultrasound stethoscope. J Am Soc Echocardiogr 2002;15:80-5.

7 Bruce CJ, Spittell PC, Montgomery SC, et al. Personal ultrasound imager: abdominal aortic aneurysm screening. J Am Soc Echocardiogr 2000;13:674-9.

8 Vourvouri EC, Poldermans D, Schinkel AFL, et al. Left ventricular hypertrophy screening using a hand-held ultrasound device. Eur Heart J 2002;26:1516-21.

9 Vourvouri EC, Poldermans D, Schinkel AFL, et al. Abdominal aortic aneurysm screening using a hand-held ultrasound device: a pilot study. Eur J Vasc Endovasc Surg 2001;22:352-4.

10 Vourvouri EC, Koroleva LY, ten Cate FJ, et al. Clinical utility and costeffectiveness of a personal ultrasound imager for cardiac evaluation during consultation rounds in patients with suspected cardiac disease. Heart 2003;89:727-30

11 Oh JK, Seward JB, Tajik AJ. The echo manual, 2nd ed. Philadelphia: Lipincott Williams and Wilkins, 1999.

12 Fleiss JL. Statistical methods for rates and proportions, 2nd ed. New York: Wiley, 1981.
13 Roelandt JRTC, Wladimiroff JW, Baars AM. Ultrasonic real time imaging with a hand-held scanner. II. Initial clinical experience. Ultrasound Med Biol 1978;4:93-7.

14 Ligtvoet $\mathrm{CM}$, Rijsterborgh $\mathrm{H}$, Kappen $\mathrm{L}$, et al. Real time ultrasonic imaging with a hand-held scanner. I. Technical description. Ultrasound Med Biol 1978;4:91-2.

15 Roelandt JRTC, Bom N, Hugenholtz P. The ultrasound cardioscope: a handheld scanner for real-time cardiac imaging. J Clin Ultrasound 1980;8:221-5.

16 Roelandt JRTC. A personal ultrasound imager (ultrasound stethoscope): a revolution in the physical cardiac diagnosis! Eur Heart J 2002;23:523-7.

17 Fedson S, Neithardt G, Thomas P, et al. Unsuspected clinically important findings detected with a small portable ultrasound device in patients admitted to a general medicine service. J Am Soc Echocardiogr 2003;16:901-5.

18 Rispler S, Rinkevich D, Markiewicz W, et al. Missed diagnosis of severe symptomatic aortic stenosis. Am J Cardiol 1995;76:728-30.

19 Galasko GIW, Lahiri A, Senior R. Portable echocardiography: an innovative tool in screening for cardiac abnormalities in the community. Eur J Echocardiogr 2003;4:119-27.

20 Rugolotto $M$, Chang CP, Hu B, et al. Clinical use of cardiac ultrasound performed with a hand-carried device in patients admitted for acute cardiac care. Am J Cardiol 2002;90:1040-2.

21 Spencer KT, Anderson AS, Bhargava A, et al. Physician-performed point-ofcare echocardiography using a laptop platform compared with physical examination in the cardiovascular patient. J Am Coll Cardiol 2001;37:2013-8

22 Quiles J, Garcia-Fernandez MA, Almeida PB, et al. Portable spectral Doppler echocardiographic device: overcoming limitations. Heart 2003;89:1014-8.

23 Seward JB, Douglas PS, Erbel R, et al. Hand-carried cardiac ultrasound (HCU) device: recommendations regarding new technology: a report from the echocardiography task force on new technology of the nomenclature and standards committee of the American Society of Echocardiography. J Am Soc Echocardiogr 2002;15:369-73.

24 Ryan T, Armstrong WF, Pearlman AD, et al. Core cardiology training in adult cardiovascular medicine (COCATS). Task force 4: training in echocardiography. J Am Coll Cardiol (in press).

25 Alexander JH, Peterson ED, Chen AY, et al. Training and accuracy of noncardiologists in simple use of point-of-care echo: a preliminary report from the Duke limited echo assessment project (LEAP). Thoraxcentre $J$ 2001;13:105-10.

26 Kimura BJ, Bocchicchio M, Willis $\mathrm{CL}$, et al. Screening cardiac ultrasonographic examination in patients with suspected cardiac disease in the emergency department. Am Heart J 2001;142:324-30.

27 Kimura BJ, Amundson SA, Willis CL, et al. Usefulness of a hand-held ultrasound device for bedside examination of left ventricular function. Am J Cardiol 2002;90:1038-9.

28 Croft LB, Cohen B, Dorantes T, et al. The echo stethoscope: is it ready for prime time by medical students? [abstract] J Am Coll Cardiol 2002;39(suppl A):448A.

29 DeCara JM, Lang RM, Koch R, et al. The use of small personal ultrasound devices by internists without formal training in echocardiography. Eur J Echocardiogr 2003;4:141-7.

\section{ELECTRONIC PAGES}

\section{Heart Online case reports: www.heartjnl.com}

$7 \mathrm{c}$ he follow electronic only articles are published in conjunction with this issue of Heart.

\section{Subacute coronary stent thrombosis in a patient developing clopidogrel associated thrombotic thrombocytopenic purpura \\ M-A von Mach, A Eich, L S Weilemann, T Münzel}

Clopidogrel, in combination with aspirin, is commonly used for the prevention of thrombosis in patients who have received coronary artery stents. As a rare but critical complication, clopidogrel associated thrombotic thrombocytopenic purpura (TTP) has previously been described. A 78 year old man presented with unstable angina and filiform subtotal stenosis of the left anterior descending artery. He was treated with balloon angioplasty and stent implantation. After four days the patient again had angina caused by stent thrombosis, which was treated with balloon angioplasty. During hospital stay the typical course of clopidogrel associated TTP was observed with thrombocytopenia and petechial purpura occurring 14 days after drug initiation and prompt response to therapeutic plasma exchanges. These findings strongly suggest that clopidogrel may have increased platelet activation and aggregation in this immunologically susceptible patient, ultimately leading to a stent thrombosis.

(Heart 2005;91:el4) www.heartjnl.com/cgi/content/full/91/ $2 / \mathrm{e} 14$

\section{Delayed severe multivessel spasm and aborted sudden death after Taxus stent implantation J W Kim, C G Park, H S Seo, D J Oh}

Sudden cardiac arrest associated with major spasm of three coronary arteries was observed about 10 hours after Taxus stent insertion in a three vessel lesion and was successfully treated by intracoronary glyceryl trinitrate infusion. This case illustrates a potential risk associated with drug eluting stent and alerts clinicians to the life threatening risk of spasm when stenting multiple vessels with drug eluting stent (especially the Taxus stent).

(Heart 2005;91:e15) www.heartjnl.com/cgi/content/full/91/ $2 / \mathrm{e} 15$ 